\title{
Rationale for Selection of Dissolution Media: Three Case Studies
}

\author{
Nikoletta Fotaki', William Brown'2, Jianmei Kochling ${ }^{3}$, \\ Hitesh Chokshi ${ }^{4}$, Hai Miao ${ }^{5}$, Kin Tang ${ }^{4}$, and Vivian Gray ${ }^{7, *}$ \\ ${ }^{6}$ V. A. Gray Consulting, Inc., 9 Yorkridge Trail, Hockessin, DE, 19707, USA
}

\section{ABSTRACT}

The selection of media in dissolution method development can sometimes be an arbitrary decision. The case studies in this article give a practical rationale that should help in selecting media, especially surfactants.

Three cases were studied: (1) the role of surfactants versus compound stability in the dissolution medium during dissolution method development, (2) the selection of a surfactant based on interactions between the dissolution medium and the drug substance, and (3) the selection of media based on formulation properties.

In the first case study, the choice of surfactant in relation to solubility and physical stability of the drug substance is shown. The second revealed an unexpected synergy between polysorbate 20 (Tween) and acetic acid solution that caused an unusual increase in the dissolution rate compared with these media used separately. In the last case study, the medium was modified by addition of surfactant to reflect a change in the formulation.

The selection of a dissolution medium should be based on drug substance and formulation characteristics as well as on interactions among components.

\section{INTRODUCTION}

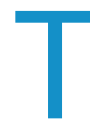

he media typically used in dissolution studies include acidic solutions, buffers, surfactants, and surfactants with acid or buffers (1). Media with bile salts and other relevant physiologically based ingredients, sometimes called biorelevant media, can be used in regulatory tests, but typically are used as research tools or for in vitro-in vivo correlation studies (2).

Surfactants are used in dissolution test methods to improve the solubility or wettability of a drug. Sometimes the decision to use a surfactant is based solely on the fact that it will facilitate drug dissolution and not on any further study. It is thus important to understand scientifically the interaction mechanisms between different types of surfactants and drug molecules as well as interactions with excipients. This understanding should guide the analyst in selecting the most appropriate media for methods that will be used in formulation development and drug product dissolution testing.

Surfactants reduce solution and surface interfacial tension by replacing water molecules on the surface (3). Surfactant molecules include two distinct components, the head (hydrophilic area) and the tail (hydrophobic area). Surfactants can be classified as anionic (e.g., sodium lauryl sulfate [SLS], also known as sodium dodecyl sulfate [SDS]), cationic (e.g., cetyl trimethyl ammonium bromide $[C T A B])$, zwitterionic (e.g., alkyl betaine), or

${ }^{*}$ Corresponding author. nonionic (e.g., Tween or Cremophor EL) (4), as shown in Figure 1.

Surfactants exist as monomers at low concentration in solutions. Aggregation occurs with increasing concentration and results in the formation of micelles. The minimum concentration of a monomer at which micelles form is called critical micelle concentration (CMC). The stability of micelles is related to their $C M C$ value: the lower the $C M C$ value of a given surfactant, the more stable the micelles (5) (Table 1). In dissolution testing, the micelle of surfactant molecules mimics the bile acid aggregates in the small intestine; the surfactant facilitates the diffusion and transport of the free solute into the bulk medium. Since dissolution is a combined effect of solubility and diffusivity, the micelle size will have an effect on the dissolution rate of molecules when different surfactants are used. Micellar-driven drug solubilization occurs with an increase in the number of micelles when the surfactant concentration is higher than the CMC value (6). In fact, solubility enhancement is a function of surfactant concentration. This relationship generally can be found for different surfactants and different compounds $(3,7,8)$.

Micellar drug solubilization is affected by many factors: the nature of the surfactant and the drug substance (e.g., nonpolar molecules are solubilized in the micellar core, whereas those with intermediate polarity are distributed along the surfactant molecules in certain intermediate positions), temperature, $\mathrm{pH}$, and ionic strength $(3,6)$. For ionic surfactants, the CMC values decrease and the micel- 


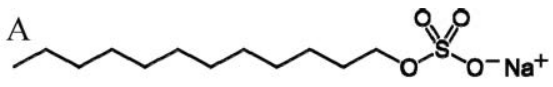

Sodium lauryl sulfate (SLS, anionic)

B $\mathrm{Br}-$<smiles>CCCCCCCCCCCCCCCC[N+](C)(C)C</smiles>

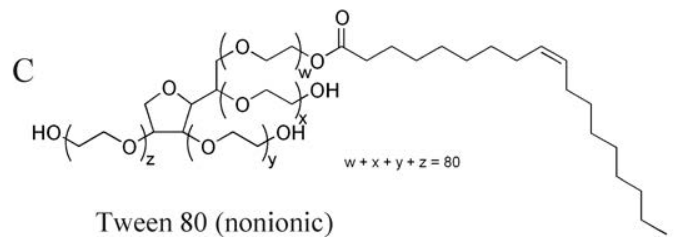

Figure 1. Structures of commonly used surfactants in dissolution: SLS (anionic), CTAB (cationic), and Tween 80 (nonionic).

lar size increases upon addition of an electrolyte to the solution. The type of surfactant determines the effect of ionic strength; for example, the solubility of mefenamic acid is affected by a change in ionic strength when SLS is used but not when CTAB is used (9). In addition, the purity of the surfactant must be considered because it can extensively affect the size and loading capacity of a micelle, which results in changes in solubility and dissolution rate $(10,11)$. Moreover, interactions among the ions and the type of surfactant used must also be considered carefully when dissolution media are selected. For example, potassium ions can interact with SLS, forming an insoluble product (12).

For acidic drugs, media containing cationic surfactants are better able to discriminate dissolution rate than media containing other types of surfactants (9).

An important point about the use of surfactants in dissolution media is that they have different effects on

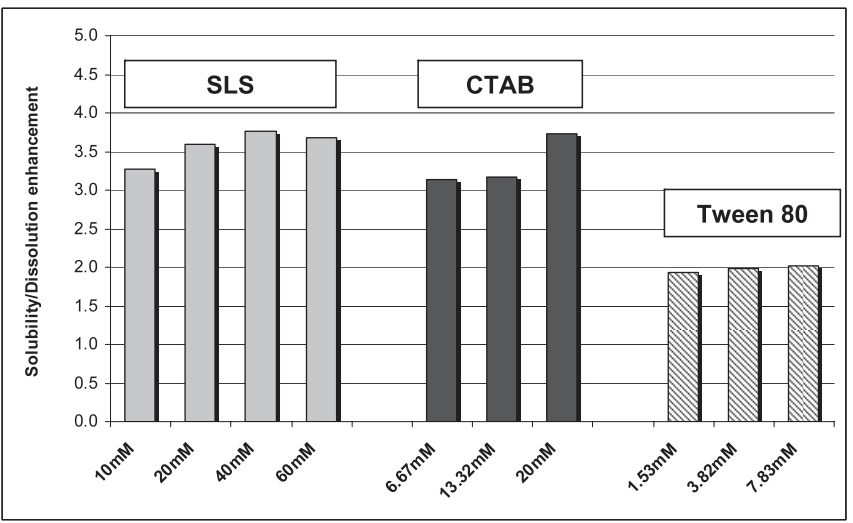

Figure 2. Solubility-dissolution enhancement of griseofulvin by ionic (SLS, CTAB) and nonionic (Tween 80) surfactants. (Data adapted and modified from ref 3.)
Table 1. CMC Values (mM) of Commonly Used Surfactants in Water at $25^{\circ} \mathrm{C}$

\begin{tabular}{lcc}
\hline Surfactant & Type & CMC (mM) \\
\hline Triton X-100 & Nonionic & 0.24 \\
\hline Tween 20 & Nonionic & 0.06 \\
\hline Tween 40 & Nonionic & 0.027 \\
\hline Tween 80 & Nonionic & 0.012 \\
\hline CTAB & Cationic & 1 \\
\hline SLS & Anionic & $7-10$ \\
\hline
\end{tabular}

the dissolution rate and solubility of the drug substance. For example, the dissolution rate of ibuprofen from tablets in $\mathrm{HCl}$ solutions with different surfactants (cationic, anionic, and nonionic) is not affected by the surfactants, even though the solubility of ibuprofen was significantly different in these solutions (9). Another example is griseofulvin, for which addition of several concentrations of ionic (SLS, CTAB) and nonionic (Tween 80) surfactants resulted in a greater enhancement of drug solubility relative to its dissolution (3). Interestingly, the enhancement of the solubility to dissolution ratio was almost constant in all cases except for the low concentration of SLS and the high concentration of CTAB (Figure 2).

In dosage systems that contain polymer, the interactions between polymers and surfactants in aqueous media give rise to the formation of association structures, thereby modifying the solution and interfacial properties. The morphologies of association complexes depend on the molecular properties of the polymer and the surfactant. The presence of a polymer lowers the CMC and reduces the size of spherical micelles $(13,14)$.

The first case study examines the choice of surfactant (anionic, cationic, or neutral) in terms of the solubility and physical stability of the drug substance. The second case study shows an unexpected synergy between polysorbate 20 (Tween) and acetic acid solution that causes an unusual increase in the dissolution rate relative to the media used

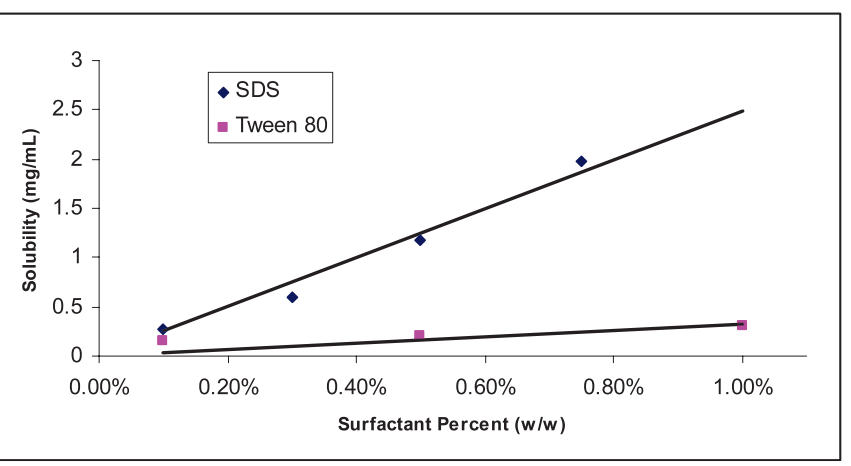

Figure 3. Solubility of Compound A in different concentrations of surfactants. 


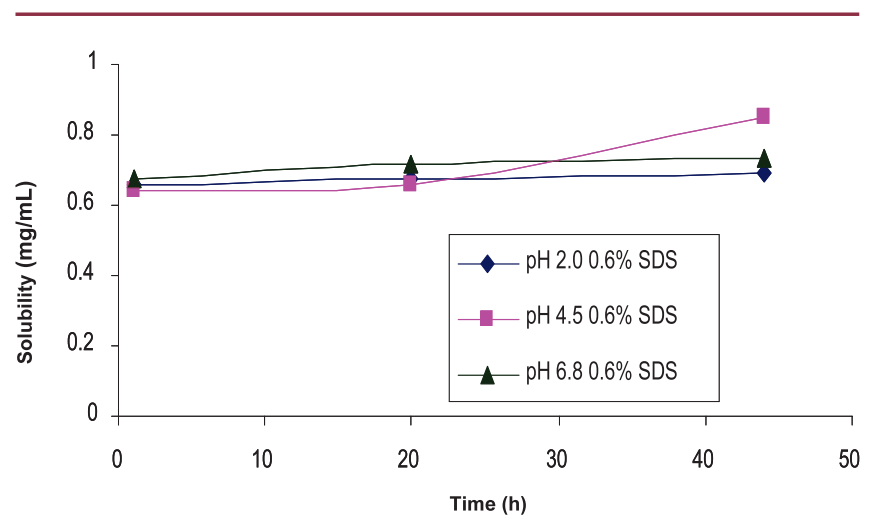

Figure 4. Stability of Compound A spray-dried dispersion (SDD) in anionic surfactant (SLS).

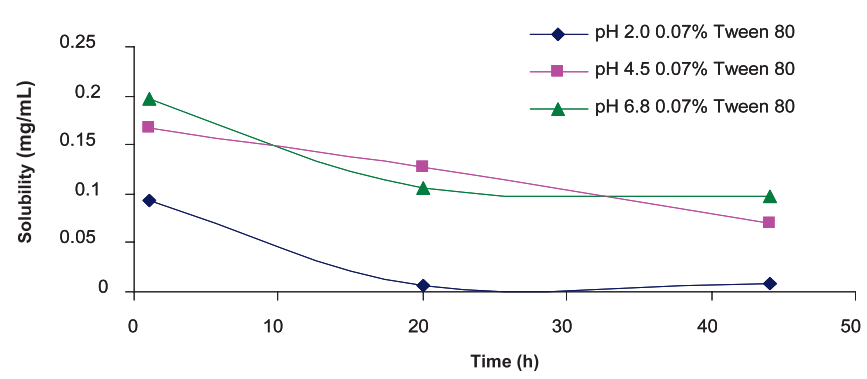

Figure 5. Stability of Compound A spray-dried dispersion (SDD) in nonionic surfactant (Tween 80 ).

separately. In the last case study, the medium was modified by addition of surfactant to reflect a change in the formulation.

\section{CASE STUDIES}

\section{Case Study 1: Effect of Different Surfactants (Anionic, Cationic, and Nonionic) on Solubility and Physical Stability}

The CMCs for SLS and Tween 80 are approximately $0.24 \%$ and $0.012 \% \mathrm{w} / \mathrm{w}$, respectively. A linear relationship exists between the surfactant (SLS or Tween 80) concentration and the equilibrium solubility of the studied compound, Compound A (Figure 3). In addition, the compound's solubility in SLS is much higher than in Tween 80 . The lower solubility in Tween 80 may be largely related to the large molecular weight of Tween 80 and the limited number of molecules that can be incorporated into the Tween 80 micelles (3). In addition, the diffusivity of Tween 80 micelles is much slower than that of SLS, which necessitates a higher Tween 80 concentration to achieve dissolution profiles that are similar to those obtained with SLS. Additionally, the preparation of a high concentration of Tween 80 dissolution medium can be a challenge. With an increase in the concentration of viscous Tween 80, solution foaming increases, which leads to large variations in analytical results.
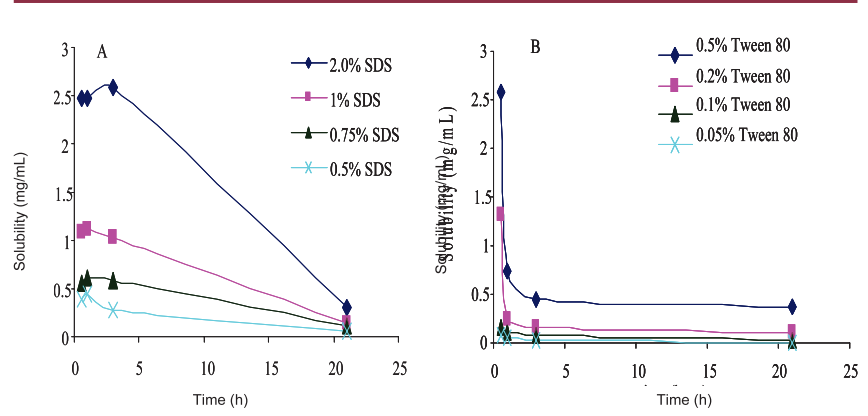

Figure 6. Stability of Compound A salt in (A) SLS and (B) Tween 80 solutions.

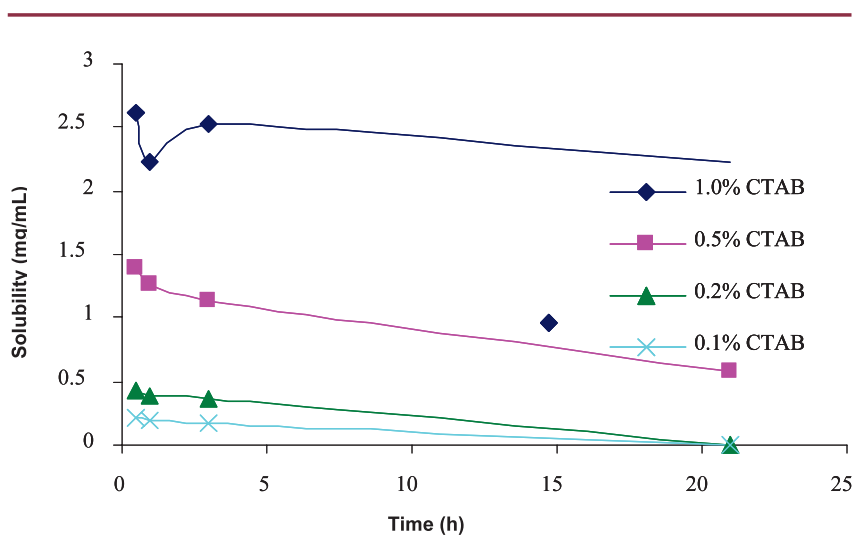

Figure 7. Solubility and stability of Compound A salt in CTAB.

Although surfactants can increase the solubility of poorly soluble compounds, different surfactants also can affect the physical stability of the study systems by maintaining the solubility of the compound in the surfactant medium without precipitation, for example. This is a common concern especially when the compound is not in the most stable polymorphic form. The roles of surfactants in compound solubility have been evaluated $(3,8)$. However, very little research has focused on the role of surfactants in the physical stability of compounds. In fact, understanding the physical stability of compounds in different surfactants is not only important for dissolution method development but is also critical for formulation development, especially liquid dosage forms. Increased understanding of surfactants and physical stability may shed light on formulation physical behavior in vivo.

The freebase of Compound $\mathrm{A}$ is a stable crystalline, neutral molecule $\left(\mathrm{p} K_{\mathrm{a}} \approx 7\right)$ that has a solubility value of about $0.2-0.4 \mu \mathrm{g} / \mathrm{mL}$ in aqueous solution in the $\mathrm{pH}$ range of $3-7$. The amorphous form was prepared by spray-drying the freebase with hypromellose acetate succinate (HPMCAS). Although changing to the amorphous spray-dried dispersion did not improve the aqueous solubility noticeably, it improved the bioavailability of the compound. A surfactant was needed to maintain sufficient solubility of the compound in $900 \mathrm{~mL}$ of dissolution medium. Several different surfactants-anionic (SLS), cationic, and neutral 


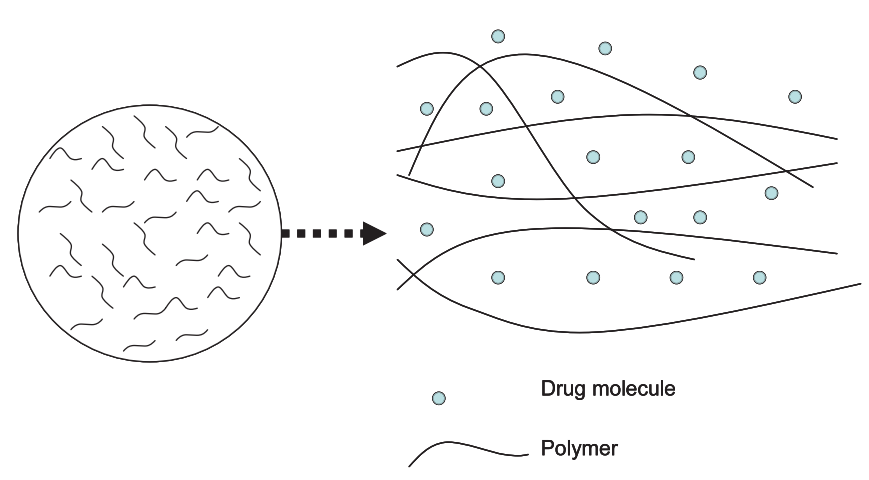

Figure 8. Example of drug-polymer solid dispersion.

(Tween 80)—were screened and differences in the physical stability of amorphous Compound A are presented in this case study.

As shown in Figure 4, amorphous Compound $A$ is stable in SLS with no change of solubility over $44 \mathrm{~h}$ in the $\mathrm{pH}$ range of 2.0-6.8. The solubility of the solid dispersion was maintained at $>0.6 \mathrm{mg} / \mathrm{mL}$ in $0.6 \% \mathrm{SLS}$, which was greater than the solubility in its freebase crystalline form.

Amorphous Compound A was added to the nonionic surfactant Tween 80 solutions in the same $\mathrm{pH}$ range of 2.0-6.8. The initial solubility was $0.2 \mathrm{mg} / \mathrm{mL}$ in $0.07 \%$ Tween 80 solution; however, solubility decreased with time, probably due to formation of crystalline forms. As shown in Figure 5, the stability of amorphous Compound A in Tween 80 also depends on $\mathrm{pH}$, and stability follows the trend of $\mathrm{pH} 1.2<4.5 \approx 6.8$.

The besylate salt form of Compound $\mathrm{A}$ also demonstrated slightly better stability in SLS solution than in Tween 80 solution. The solution was stable for $3.5 \mathrm{~h}$ in SLS versus $<1$ hour stability in Tween 80 , as shown in Figure 6 . In addition to noting solubility changes as an exhibition of physical stability, physical form change was detected using X-ray powder diffraction (XRPD) (data not shown). The original polymorph was detected in a precipitate only at the 1-h time point in SLS solution and not at later time points ( $4 \mathrm{~h}$ and $22 \mathrm{~h}$ ) in Tween 80 . Compound A salt dissolved in a cationic surfactant (CTAB) had stability similar to that in SLS solution (Figure 7) and was physically stable only for $1 \mathrm{~h}$. Other forms were detected by XRPD at 4 and $22 \mathrm{~h}$.

\section{Discussion}

In the past few years, spray-dried solid dispersion (SDD) processing has gained popularity in the pharmaceutical industry (15). This procedure is used to enhance the bioavailability of poorly soluble drugs by converting a stable crystalline form, which has low solubility, to a metastable amorphous form that has higher solubility. However, even with form conversion, solubility enhancers such as surfactants are still needed, sometimes in both the formulation and the dissolution media.

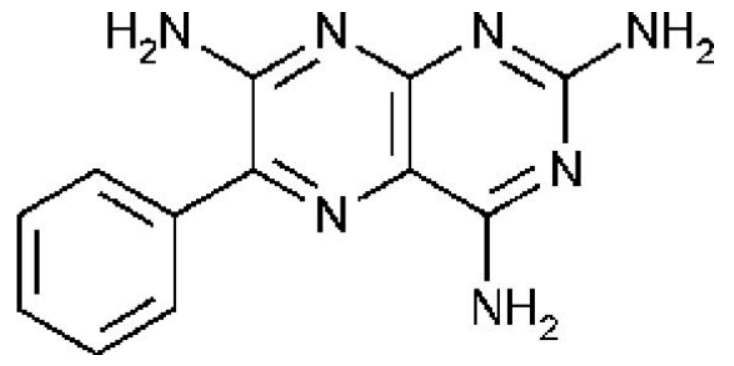

Figure 9. Chemical structure of triamterene.

Figure 8 illustrates a drug-polymer solid dispersion in which the polymer serves as a carrier and the drug molecule is dissolved in the polymer matrix (16). The drying process uses a volatile solvent in which both polymer and drug molecules dissolve and generate an amorphous form of the drug molecule. The copresence of polymer and drug molecule in the solid mixture not only increases drug solubility but also the stability of the amorphous drug. The increased solubility is caused by easier accessibility for the water molecules, and the greater wettability of the drug molecules is caused by the use of a water-soluble polymer and sometimes a surfactant. The increased stability of the SDD system may be due to extra energy (and thus more time) needed for the drug molecules to separate from the polymer matrix. Some spray-dried dispersions can be stable for years at room temperature $(17,18)$, although manufacturers should take care to treat such systems as amorphous by protecting them from moisture.

The dissolution medium has a large influence on the solubility and stability of amorphous drug substance molecules. Using the HPMCAS solid dispersion compound as an example, we see that the stability of the solid dispersion system in solution depends largely on the $\mathrm{pH}$ and the surfactant type (19) (Figures 4 and 5).

As shown in Figure 4, Compound $A$ was stable in the SLS solution for more than $40 \mathrm{~h}$ but was not stable in the polysorbate 80 solution (Figure 5 ). The stability of Compound $A$ in the SLS solution may be related to the interaction between the polymer and the surfactant micelle hydrophobic chain $(20,21)$. This solid dispersion also interacted with the Tween 80 polymer chain due to the small number of drug molecules that can be incorporated into the Tween 80 micelles and low diffusivity of the micelles (3). However, the lower solubility of Compound A solid dispersion in Tween 80 may offer a greater chance for Compound $A$ to be in a free form in solution. Once in solution, the polymer and Compound A separated, which resulted in the conversion of the amorphous form to its stable crystalline freebase form. A comparison of Figures 4 and 5 shows that the conversion rate of the amorphous to the crystalline form was so low that it was not observed within $44 \mathrm{~h}$ in SLS, but the conversion was almost immediate in the Tween 80 solution (Figure 5). 


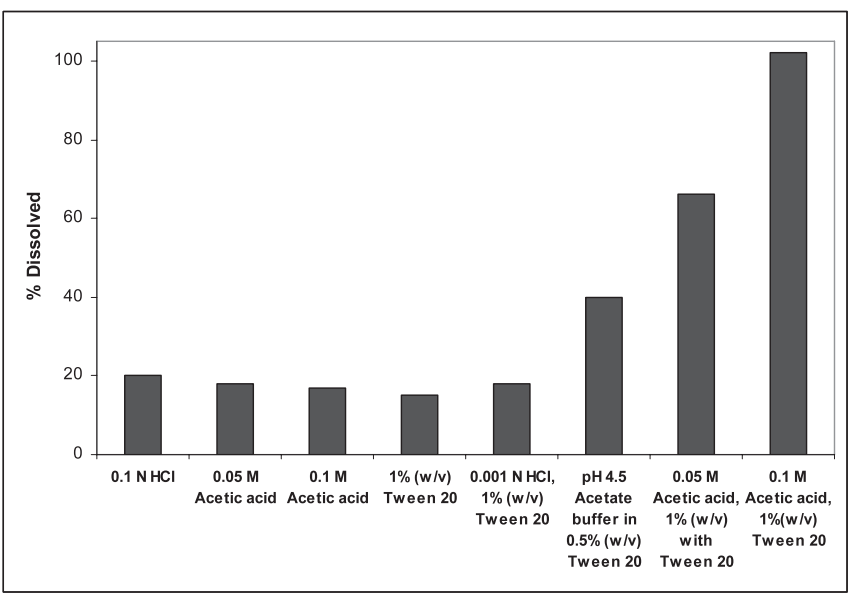

Figure 10. Dissolution results at $2 \mathrm{~h}$ for triamterene in various media (\% label claim).

Interestingly, the Compound A salt form had similar stability in both anionic and cationic surfactant solutions, as shown in Figures $6 \mathrm{~A}$ and 7. The stability of the compound/ surfactant solution systems was probably dictated by the similar micelle size and the number of drug molecules that were incorporated into the SLS and CTAB micelles rather than by the surfactant charge state. The results are consistent with observations reported by Balakrishnan et al. (3).

\section{Case Study Conclusions}

As for choosing a surfactant for the dissolution method, there are a few scenarios. If the surfactant is used to increase the solubility of the compound and there is no concern about physical stability, decisions can be based simply on the enhancement of solubility, and both ionic and nonionic can be considered. However, if there is a physical stability issue, the type of surfactant that allows slower rate of conversion due to micelle size and the number of drug molecules that can be incorporated into the micelles (e.g., SLS and CTAB) may have an advantage. A final and important consideration is chemical stability. Although any degradation $<1 \%$ is not a major concern, degradation of API in the surfactant solution should also be considered during dissolution method development.

\section{Case Study 2: Synergistic Effect of Surfactant and Acetic Acid Solution}

An improved dissolution method for an immediaterelease (IR) combination capsule product of hydrochlorothiazide and triamterene $\left(\mathrm{p} K_{\mathrm{a}}=6.2 ;\right.$ Figure 9$)$ was developed (22). The dissolution performance of the less soluble component, triamterene, in a series of media was evaluated (Figure 10). A five-fold increase in triamterene percent dissolved was observed in the selected medium ( $0.1 \mathrm{M}$ acetic acid with $1 \% \mathrm{w} / \mathrm{v}$ Tween 20 ) relative to the percent dissolved in each medium used separately $(0.1$ $\mathrm{M}$ acetic acid or $1 \%(\mathrm{w} / \mathrm{v})$ with Tween 20$)$. The dissolution

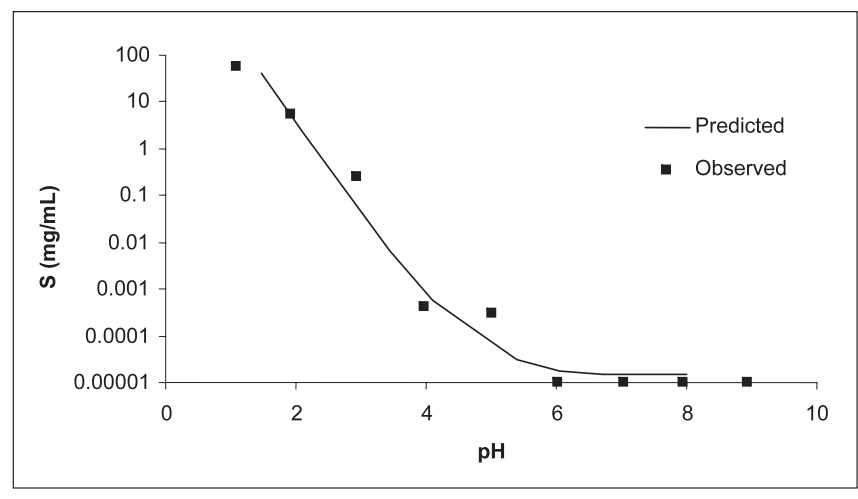

Figure 11. $\mathrm{pH}$-solubility profile of compound $B$.

conditions were USP Apparatus 1, rotating basket, 100 rpm, and $900 \mathrm{~mL}$ of medium at $37^{\circ} \mathrm{C}$.

\section{Discussion}

The $0.1 \mathrm{~N} \mathrm{HCl}$ medium resulted in $20 \%$ triamterene dissolved from the product in $2 \mathrm{~h}$ (Figure 10). At $2 \mathrm{~h}$, the percent dissolved was $<1 \%$ in both $0.5 \%$ SLS and $0.5 \%$ SLS adjusted to $\mathrm{pH} 4.5$ with acetate buffer ( $0.02 \mathrm{M}$ sodium acetate and $0.03 \mathrm{M}$ acetic acid).

Complete dissolution was observed in the medium 0.1 $M$ acetic acid with 1\% Tween 20 (Figure 10). The increased triamterene dissolution rate apparently was a product of synergetic interaction between the Tween 20 and acetic acid. The $\mathrm{pH}$ value of this medium was 3.2. The increased triamterene dissolution is not attributable to medium $\mathrm{pH}$, Tween 20 concentration, or the simple combination of $\mathrm{pH}$ with Tween 20 concentration. A 5- to 6-fold increase in dissolution was obtained over $1 \%$ Tween $20,0.1 \mathrm{M}$ acetic acid, and $0.001 \mathrm{~N} \mathrm{HCl}$ with $1 \%$ Tween 20. There was an incremental increase in the dissolution rate with increasing concentration of acetic acid from $0.05 \mathrm{M}$ to $0.1 \mathrm{M}$ with 1\% Tween 20.

The effect of the presence of surfactant on the dissolution rate in this case was observed only with the nonionic surfactant (Tween 20) and not with the anionic one (SLS). On the one hand, triamterene is weak base, and hence solubility should increase with a decrease in $\mathrm{pH}$. On the other hand, SLS has a higher ionization with an increase in $\mathrm{pH}\left(\mathrm{p} K_{\mathrm{a}}=1.9\right)$, which should result in a favorable situation for solubility enhancement. The anions of SLS may interact with the cationic drug species to form an insoluble salt and result in desolubilization; this phenomenon has been reported $(8,23)$. The better solubilization obtained with the nonionic surfactant could be because nonionic surfactants provide high micellar concentration and good molar solubilization capacity due to the low CMC value (8).

\section{Case Study Conclusions}

The interaction of acetic acid with Tween 20 produced an increase in the dissolution rate of the limiting com- 
ponent, triamterene, that was not attributable to the surfactant concentration or the $\mathrm{pH}$ acting alone. Nor was the concentration of acetic acid solely attributable as a factor. Apparently, the presence of acetic acid enhances the dissolution rate of triamterene in Tween 20 medium. This example suggests that surfactant must be chosen carefully when $\mathrm{pH}$ also has an effect on API solubility.

\section{Case Study 3: Selection of Dissolution Medium Based on Formulation Change}

A successful dissolution method was developed for an IR formulation of a dibasic compound (Compound B). The dissolution conditions were USP Apparatus 2 (rotating paddle), $100 \mathrm{rpm}$, and $900 \mathrm{~mL}$ of $0.003 \mathrm{~N} \mathrm{HCl}(\mathrm{pH} 2.5)$ at $37^{\circ} \mathrm{C}$. A change in the formulation necessitated the change of the dissolution method to a medium of $\mathrm{pH} 6.8$ with the addition of surfactant (SLS).

\section{Discussion}

Compound $\mathrm{B}$ is a high molecular weight dibasic compound $\left(\mathrm{p} K_{a 1}=3.93, \mathrm{p} K_{a 2}=5.42\right)$. The freebase was used in the development of an IR tablet to be taken once or twice a day. The compound is highly soluble in acidic aqueous media and is sparingly soluble in aqueous media at a $\mathrm{pH}$ greater than 4, as shown in the $\mathrm{pH}$-solubility profile in Figure 11. Based on the high solubility observed at acidic $\mathrm{pH}$, a dissolution test was developed using USP Apparatus 2 (paddles) operated at $75 \mathrm{rpm}$ with $900 \mathrm{~mL}$ of $0.003 \mathrm{~N} \mathrm{HCl}$ ( $\mathrm{pH}$ 2.5). The amount of drug dissolved, determined by UV, is shown in Figure 12 for three 250-mg tablet formulations. Tablet $A$ and Tablet $B$ are of the same prototype composition and differ only in the particle size of the drug substance used to prepare the tablet $\left(\mathrm{A}: D_{50}=217 \mu \mathrm{m}\right.$; $\left.\mathrm{B}: D_{50}=4.6 \mu \mathrm{m}\right)$. The composition of Tablet $C\left(D_{50}=7.3\right.$ $\mu \mathrm{m})$, which differs slightly from that of Tablets $A$ and $B$, contains a higher drug load to obtain a smaller tablet for patient compliance. As shown in Figure 12, differences in dissolution rate due to the pronounced differences in drug substance particle size can be seen in the acidic media. Therefore, particle size reduction is desired to obtain a rapidly dissolving product in acidic media. It is not clear if the differences in dissolution rate between Tablet $B$ and Tablet $C$ are attributable to particle size, as there are also formulation differences. The higher drug load of a weakly basic compound that exhibits high solubility at acidic $\mathrm{pH}$ may drive the slightly faster dissolution rate observed with Tablet C.

An in vivo study in dogs revealed differences consistent with those observed in the in vitro study (Figure 13), supporting the selection of the dissolution method.

A second-generation formulation containing the drug and an anionic polymer in the tablet matrix was developed. Because the anionic polymer is insoluble in acidic solution, the original dissolution method was not suitable. Figure 14A shows the dissolution profiles for the original 250-mg IR tablet (Formulation I) and a 50-mg IR tablet

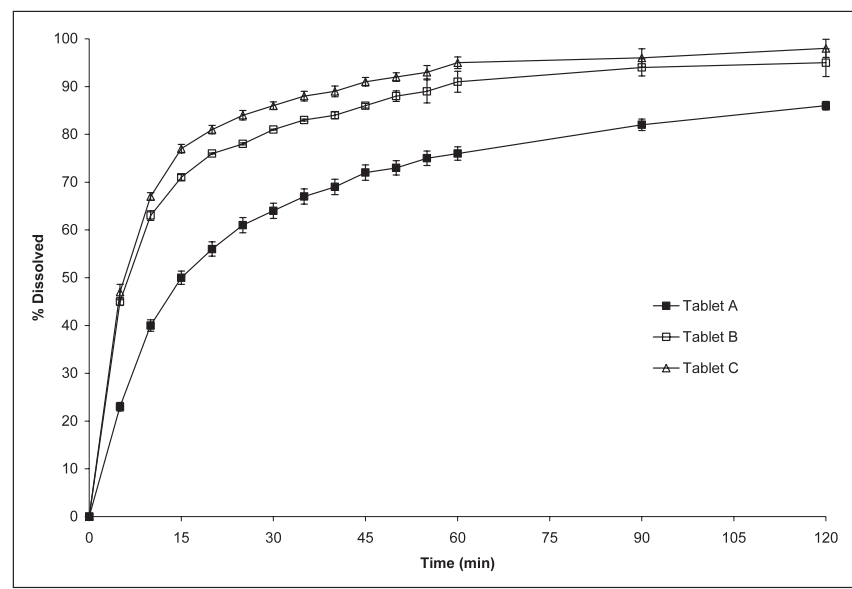

Figure 12. Dissolution profile of compound B, 250-mg film-coated IR tablets. Tablet A: Prototype \#1 tablet made with unmilled drug substance $\left(D_{50}=217 \mu \mathrm{m}\right)$; Tablet B: Prototype \#1 tablet made with micronized drug substance $\left(D_{50}=4.6 \mu \mathrm{m}\right)$; Tablet $C$ : Prototype \#2 tablet made with micronized drug substance $\left(D_{50}=7.3 \mu \mathrm{m}\right)$. Testing performed with USP Apparatus 2, $75 \mathrm{rpm}, 900 \mathrm{~mL}$ of $0.003 \mathrm{~N} \mathrm{HCl}$; detection by UV.

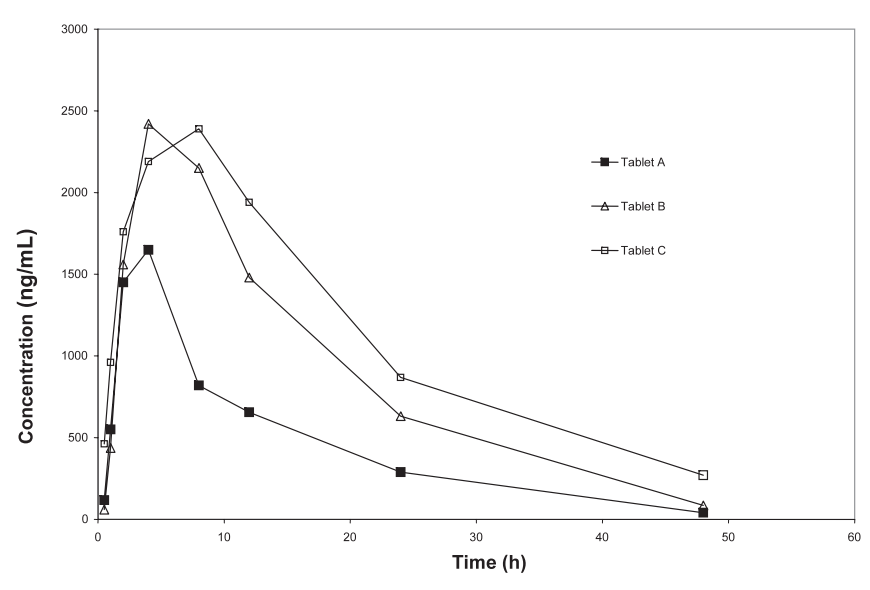

Figure 13. Plasma concentration profile of compound $B$ dosed to dogs as 250-mg film-coated IR tablets. Tablet A: Prototype \#1 tablet made with unmilled drug substance $\left(D_{50}=217 \mu \mathrm{m}\right)$; Tablet B: Prototype \#1 tablet made with micronized drug substance $\left(D_{50}=4.6 \mu \mathrm{m}\right)$; Tablet $C$ : Prototype $\# 2$ tablet made with micronized drug substance $\left(D_{50}=7.3 \mu \mathrm{m}\right)$.

containing the anionic polymer (Formulation II). In acidic medium, Formulation I exhibits a rapid dissolution profile, whereas Formulation II exhibits a considerably slower profile. To address the $\mathrm{pH}$-dependent solubility of the anionic polymer in Formulation II, the $\mathrm{pH}$ of the medium was raised to 6.8 where the anionic polymer will dissolve. A small amount of SLS (0.25\%) was added to the new medium to solubilize the weak base drug in Formulation II. Figure 14B shows the dissolution profiles for the same two tablet formulations. Both Formulation I and Formulation II exhibit dissolution profiles that are consistent with an IR tablet product. The slightly lower rate and extent of dissolution observed in Formulation I are attributed to the 
A

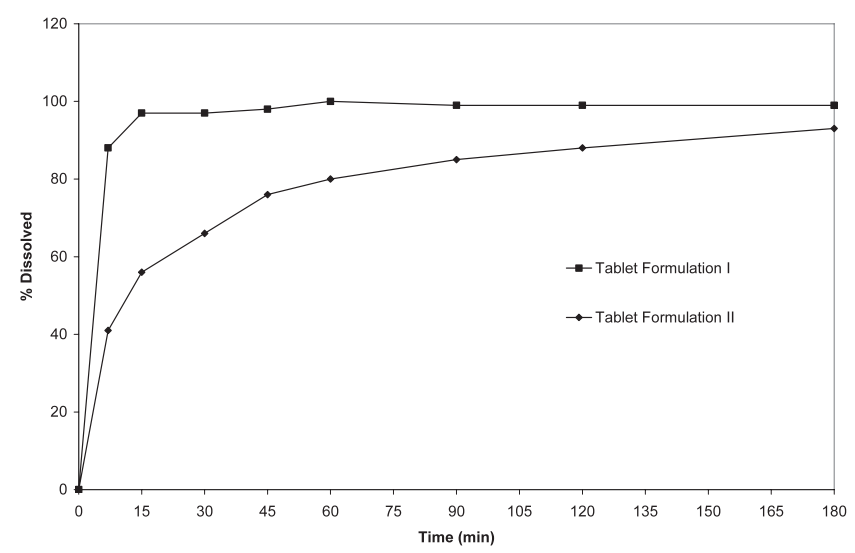

B

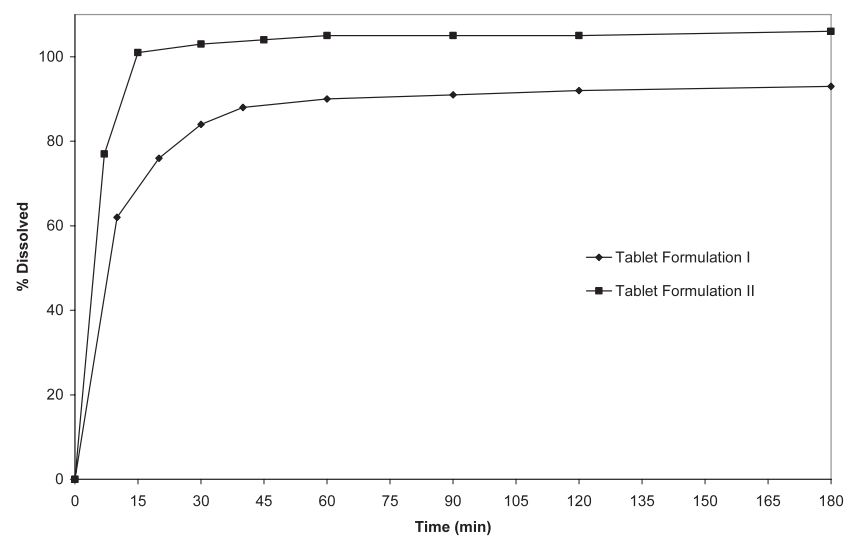

Figure 14. Dissolution profiles obtained in (A) $0.01 \mathrm{~N} \mathrm{HCl}$ and (B) $0.25 \%$ sodium lauryl sulfate/ $\mathrm{pH} 6.8$ phosphate buffer for Tablet Formulation I (IR tablet made with conventional excipients) and Tablet Formulation II (IR tablet containing anionic polymer). Testing performed with USP Apparatus 2, $75 \mathrm{rpm}, 900 \mathrm{~mL}$ medium; detection by UV.

higher dosage strength, 250 mg, versus 50 mg for Formulation II.

\section{Case Study Conclusions}

In this example, an acidic medium was used for the dissolution method of a weak base. A formulation change with the inclusion of an anionic polymer in the matrix necessitated the change to a more alkaline dissolution medium, and thus a surfactant was selected to enable solubilization of the API. This study demonstrates the role of formulation excipients in dissolution method development. Decisions on dissolution method development must be based not only on API properties but also on formulation properties. The dissolution method may need to be changed substantially due to formulation excipient properties.

\section{CONCLUSIONS}

The case studies described in this paper present a rationale for the selection of dissolution media. Each case provides instructions on ways to choose media that are consistent with a good understanding of the product tested. In summary, product solubility, product stability, synergistic effects of particular agents, and changes in the formulation and formulation excipients are essential factors to consider in the selection of an appropriate dissolution medium.

\section{REFERENCES}

1. The United States Pharmacopeia and National Formulary USP 32-NF 27; The United States Pharmacopeial Convention, Inc.: Rockville, MD, 2009.

2. Fotaki, N.; Vertzoni, M. Biorelevant Dissolution Methods and Their Applications in In Vitro-In Vivo Correlations for Oral Formulations. The Open Drug Deliv. J. 2010, 4, 2-13.

3. Balakrishnan, A.; Rege, B. D.; Amidon, G. L.; Polli, J. E. Surfactant-mediated dissolution: contributions of solubility enhancement and relatively low micelle diffusivity. J. Pharm. Sci. 2004, 93 (8), 2064-2075.

4. Fathi Azarbayjani, A.; Jouyban, A.; Chan, S. Y. Impact of surface tension in pharmaceutical sciences. J. Pharm. Pharm. Sci. 2009, 12 (2), 218-228.

5. Florence, A. T.; Attwood, D. Surfactants. In Physicochemical Principles of Pharmacy, 4th ed.; Pharmaceutical Press: London, 2006; pp 177-228.

6. Rangel-Yagui, C. O.; Pessoa, A. Jr.; Tavares, L. C. Micellar solubilization of drugs. J. Pharm. Pharm. Sci. 2005, 8 (2), 147-163.

7. Morozowich, W.; Gao, P.; Charton, M. Speeding the development of poorly soluble/poorly permeable drugs by SEDDS/S-SEDDS formulations and prodrugs. Part 1. Am. Pharm. Rev. 2006, 9, 110-114.

8. Chakraborty, S.; Shukla, D.; Jain, A.; Mishra, B.; Singh, S. Assessment of solubilization characteristics of different surfactants for carvedilol phosphate as a function of pH. J. Colloid Interface Sci. 2009, 335 (2), 242-249.

9. Park, S. H.; Choi, H. K. The effects of surfactants on the dissolution profiles of poorly water-soluble acidic drugs. Int. J. Pharm. 2006, 321 (1-2), 35-41.

10. Crison, J. R.; Shah, V. P.; Skelly, J. P.; Amidon, G. L. Drug dissolution into micellar solutions: development of a convective diffusion model and comparison to the film equilibrium model with application to surfactantfacilitated dissolution of carbamazepine. J. Pharm. Sci. 1996, 85 (9), 1005-1011.

11. Qiang, D.; Gunn, J. A.; Schultz, L.; Li, Z. J. Evaluation of the impact of sodium lauryl sulfate source variability on solid oral dosage form development. Drug Dev. Ind. Pharm. 2010, 36 (12), 1486-1496.

12. Ropers, M. H.; Czichocki, G.; Brezesinski, G. Counterion effect on the thermodynamics of micellization of alkyl sulfates. J. Phys. Chem. B 2003, 107 (22), 5281-5288.

13. Benrraou, M.; Bales, B.; Zana, R. Effect of the nature of the counterion on the interaction between cesium and tetraalkylammonium dodecylsulfates and 
poly(ethylene oxide) or poly(vinylpyrolidone). J. Colloid Interface Sci. 2003, 267 (2), 519-523.

14. Nagarajan, R. Polymer-Surfactant Interactions. In Detergents for the New Millennium, Proceeding of New Horizons Conference, American Oil Chemists Society and Consumer Specialty Products Association, Fort Myers, FL, Oct 14-17, 2001.

15. Bikiaris, D. N. Solid dispersions, part I: recent evolutions and future opportunities in manufacturing methods for dissolution rate enhancement of poorly watersoluble drugs. Expert Opin. Drug Delivery 2011, 8 (11), 1501-1519.

16. Tang, L.; Khan, S. U.; Muhammad, N. A. Evaluation and selection of bio-relevant dissolution media for a poorly water-soluble new chemical entity. Pharm. Dev. Technol. 2001, 6 (4), 531-540.

17. Shah, V. P.; Konecny, J. J.; Everett, R. L.; McCullough, B.; Noorizadeh, A. C.; Skelly, J. P. In vitro dissolution profile of water-insoluble drug dosage forms in the presence of surfactants. Pharm. Res. 1989, 6 (7), 612-618.

18. Friesen, D. T.; Shanker, R.; Crew, M.; Smithey, D. T.; Curatolo, W. J.; Nightingale, J. A. Hydroxypropyl methylcellulose acetate succinate-based spray-dried dispersions: an overview. Mol. Pharm. 2008, 5 (6), 1003-1019.

19. Curatolo, W.; Nightingale, J. A.; Herbig, S. M. Utility of hydroxypropylmethylcellulose acetate succinate (HPMCAS) for initiation and maintenance of drug supersaturation in the GI milieu. Pharm. Res. 2009, 26 (6), 1419-1431.

20. Bai, G.; Catita, J. A. M.; Nichifor, M.; Bastos, M. Microcalorimetric evidence of hydrophobic interactions between hydrophobically modified cationic polysaccharides and surfactants of the same charge. J. Phys. Chem. B 2007, 111 (39), 11453-11462.

21. de Martins, R. M.; Silva, C. A.; Becker, C.; Samios, D.; Bica, C. I. D.; Christoff, M. Studies on Anionic Surfactant Structure in the Aggregation with (Hydroxypropyl)cellulose. Polimeros 2002, 12 (2), 109-114.

22. Albert, A. Selective Toxicity, 4th ed.; Methuen: London, 1968.

23. Jain, A.; Ran, Y.; Yalkowsky, S. Effect of pH-sodium lauryl sulfate combination on solubilization of PG-300995 (an anti-HIV agent): a technical note. AAPS PharmSciTech 2004, 5 (3), 65-67.

24. Sigma-Aldrich: Detergents Properties and Applications. http://www.sigmaaldrich.com/img/assets/15402/ Detergent_Selection_Table.pdf (accessed June 19, 2013).

25. Hait, S. K.; Moulik, S. P. Determination of critical micelle concentration (CMC) of nonionic surfactants by donoracceptor interaction with iodine and correlation of CMC with hydrophile-lipophile balance and other parameters of the surfactants. J. Surfactants Deterg. 2001, 4 (3), 303-309. 